\title{
Distribución de estadios de OLGA y OLGIM según edad y estado del Helicobacter pylori en un hospital público nivel III en Lima, Perú
}

\author{
Andrea Carlin Ronquillo, ${ }^{1}$ Alex Ventura León, ${ }^{2}$ Jorge L Espinoza Ríos, ${ }^{3}$ Eduar A Bravo Paredes, ${ }^{3}$ \\ Paúl Gómez Hinojosa, ${ }^{1}$ Shirley Alva Solis, ${ }^{2}$ José L Pinto Valdivia, ${ }^{3}$ Wilmer Silva-Caso ${ }^{4}$ \\ ${ }^{1}$ Médico asistente de Patología, Hospital Cayetano Heredia. \\ ${ }^{2}$ Médico residente de Gastroenterología, Hospital Cayetano Heredia. \\ ${ }^{3}$ Médico asistente de Gastroenterología, Hospital Cayetano Heredia. Profesor de la Facultad de Medicina "Alberto Hurtado"- Universidad Peruana \\ Cayetano Heredia. \\ ${ }^{4}$ Escuela de Medicina, Centro de Investigación e Innovación de la Facultad de Ciencias de la Salud, Universidad Peruana de Ciencias Aplicadas. \\ Facultad de Ciencias de la Salud, Universidad Tecnológica del Perú. \\ Lima, Perú.
}

Acta Gastroenterol Latinoam 2021;51(1):76-83

Recibido: 20/05/2019 / Aceptado: 16/02/2021 / Publicado online: 22/03/2021 / https://doi.org/10.52787/uccm2971

\section{Resumen}

Introducción. El sistema operativo de evaluación de gastritis (OLGA) y el enlace operativo sobre la evaluación de metaplasia intestinal gástrica (OLGIM) proporcionan una evaluación del riesgo de desarrollar cáncer gástrico. Objetivo. Evaluar la distribución de estadios del OLGA y OLGIM por edad y presencia del Helicobacter pylori. Material y métodos. Se estudiaron 197 sujetos sometidos a una endoscopia digestiva alta electiva. La presencia del H. pylori y los cambios histológicos se evaluaron utilizando el sistema actualizado de Sídney. Los estadios III y IV del OLGA/OLGIM se consideraron estadios de alto riesgo. Resultados. La tasa del H. pylori fue del 56,85\% (112/197). Los casos de OLGA/OLGIM de alto riesgo fueron poco frecuentes: 7/112 (6,5\%) casos del OLGA en el grupo del H. pylori posi-

Correspondencia: Andrea Carlin Ronquillo

Tiziano 1070, San Borja. Lima, Perú

Tel.: 0051988869184

Correo electrónico: andrea.carlinr@gmail.com tivo y $6 / 85(7 \%)$ en el del $H$. pylori negativo; $5(4,4 \%)$ casos del OLGIM en el H. pylori positivo y $6(7 \%)$ en el $H$. pylori negativo. La proporción de estadios avanzados del OLGA y OLGIM aumentó con la edad $(p<0,001)$. No se encontró el OLGA de alto riesgo antes de los 40 años independientemente de la presencia del $H$. pylori, pero aumentó a 16,2\%, $10,3 \%, 17,3 \%$ y 40,8\% en sujetos en la cuarta, quinta, sexta y séptima década de vida respectivamente. El OLGIM de alto riesgo mostró una tendencia similar: $0 \%$ antes de los 40 años y hasta 22,6\% en personas de 70 años. Conclusiones. Los casos del OLGA/OLGIM de alto riesgo son infrecuentes antes de los 40 años y aumentan significativamente con la edad. No se evidenció relación con la presencia del H. pylori. Según estos protocolos solamente la quinta parte de los pacientes requeriría estrictamente un control endoscópico.

Palabras claves. Gastritis atrófica, sistema operativo de evaluación de gastritis (OLGA), enlace operativo sobre la evaluación de metaplasia intestinal gástrica (OLGIM), Helicobacter pylori, edad.

OLGA and OLGIM Stage Distribution according to Age and Helicobacter pylori Status in a Public Hospital in Lima, Peru

\section{Summary}

Introduction. The operative link for gastritis assessment $(O L G A)$ and the operative link on gastric intestinal meta- 
plasia assessment (OLGIM) staging systems have been suggested to provide risk of assessment for gastric cancer. Objective. To evaluate the distribution of OLGA and OLGIM staging by age and Helicobacter pylori status. Material and methods. We studied 197 subjects undergoing elective upper gastrointestinal endoscopy. The presence of the H. pylori and histological changes were evaluated using the updated Sydney system. Stages III and IV of OLGA/OLGIM were considered high risk stages. Results. The $H$. pylori rate was $56.85 \%$ (112/197). High-risk OLGA/OLGIM cases were rare: $7 / 112(6.5 \%)$ cases of OLGA in the H. pylori positive group and $6 / 85$ (7\%) in the H. pylori negative group; 5 (4.4\%) cases of OLGIM in the H. pylori positive and $6(7 \%)$ in the $H$. pylori negative. The proportion of advanced stages of OLGA and OLGIM increased with age $(p<0.001)$. Highrisk OLGA was not found before age 40 regardless of the presence of $H$. pylori, but increased to 16.2\%, 10.3\%, 17.3\% and $40.8 \%$ in subjects in the fourth, fifth, sixth and seventh decade of life respectively. The OLGIM high risk showed a similar trend: $0 \%$ before 40 years and up to $22.6 \%$ in people of 70 years. Conclusions. High-risk OLGA/OLGIM cases are infrequent before age 40 and increase significantly with age. No relation was found with the presence of the H. pylori. According to these protocols, only a fifth of the patients would strictly require endoscopic control.

Keywords. Atrophic gastritis, Operative System of Evaluation of Gastritis (OLGA), Operative Connection on the Evaluation of Gastric Intestinal Metaplasia (OLGIM) Helicobacter pylori, age.

\section{Abreviaturas}

OLGA: Sistema operativo de evaluación de gastritis.

OLGIM: Enlace operativo sobre la evaluación de metaplasia intestinal gástrica.

CG: Cáncer gástrico.

GA: Gastritis atrófica.

MI: Metaplasia intestinal.

EDA: Endoscopía digestiva alta.

$D E$ : Desviación estándar.

OR: Odds ratio.

\section{Introducción}

La incidencia del cáncer gástrico (CG) disminuyó en las últimas décadas. Hace 40 años era más común a nivel mundial y, actualmente, ocupa el quinto lugar en frecuencia y es la tercera causa de muerte por cáncer. ${ }^{1,2}$ Asimismo, en Lima, es la segunda neoplasia más común y la primera causa de muerte por cáncer. ${ }^{3}$ La mayoría de los casos se produce en los países en vías de desarrollo debido a los malos hábitos higiénico- dietéticos y la mayor prevalencia del Helicobacter pylori (H. pylori). ${ }^{1-5}$

Es importante mencionar que la disminución en la incidencia se ha interrumpido y la población joven está siendo más afectada por razones no conocidas. ${ }^{6}$ Además, por el aumento de la expectativa de vida, el número absoluto de casos nuevos anuales está creciendo. ${ }^{4,6}$ Entonces, el CG continúa siendo un serio problema de salud pública y es importante entender la patogénesis para establecer las medidas de prevención.

El adenocarcinoma gástrico de tipo intestinal, según la clasificación de Lauren, tiene factores de riesgo bien establecidos como la presencia de la gastritis atrófica (GA), la metaplasia intestinal (MI) y la displasia de la mucosa gástrica. ${ }^{7,8}$ Esta secuencia de lesiones premalignas fue descripta por Pelayo Correa en la década de los setenta. ${ }^{5,9} \mathrm{Al}$ mismo tiempo, la literatura señala que la extensión de la gastritis y de la GA se relaciona directamente con el riesgo de desarrollar cáncer. ${ }^{10-12}$

La división histológica para biopsias gástricas más utilizada es el sistema de Sídney modificado, cuyo reporte final combina el tipo, la intensidad y la extensión de la patología gástrica, además de la posible etiología. ${ }^{13,14}$ También provee una escala visual análoga para clasificar el grado de la inflamación, la atrofia, la metaplasia y la densidad del $H$. pylori. ${ }^{14}$ Sin embargo, este método carece de valor pronóstico para el CG. ${ }^{15}$

En el año 2005, el grupo internacional Operative Link on Gastritis Assessment (OLGA) planteó un nuevo sistema de clasificación para describir los estadios de la gastritis que van del 0 al IV y ya se ha demostrado que los pacientes con estadios avanzados (III y IV) tienen mayor riesgo de CG. ${ }^{15-23}$ Por la variabilidad en el diagnóstico de GA, se ha sugerido reemplazar la GA por MI (sistema OLGIM) debido a una fuerte correlación positiva con el OLGA y su mayor acuerdo interobservador. ${ }^{24}$ La prevalencia de la GA y la MI aumenta con la edad avanzada y la progresión histológica se debe principalmente a una infección por el H. pylori de larga duración. ${ }^{25-28}$

El objetivo del estudio es evaluar la distribución de los estadios del OLGA y OLGIM por edad y presencia del H. pylori en nuestra población. Es relevante porque identificar precozmente las lesiones premalignas permitiría detener la progresión al carcinoma ${ }^{7,8}$ y conocer la distribución de estas lesiones ayuda a plantear medidas de prevención pertinentes.

\section{Material y métodos}

Se realizó un estudio prospectivo descriptivo para determinar la frecuencia de los estadios avanzados de la GA y 
la MI en los pacientes a los cuales se les realizó una endoscopía digestiva alta (EDA) electiva en un hospital público de nivel III en Lima (Perú). Se estudiaron 197 sujetos sometidos a una EDA electiva de manera consecutiva. La presencia del $H$. pylori y los cambios histológicos fueron evaluados por un patólogo experto de nuestro hospital utilizando el sistema actualizado de Sídney. Los estadios III y IV del OLGA/OLGIM se consideraron estadios de alto riesgo.

La población de estudio corresponde a todos los pacientes sometidos a una endoscopía electiva en el servicio de Gastroenterología del Hospital Cayetano Heredia en el periodo de octubre a diciembre del año 2017 y que durante el procedimiento endoscópico se obtuvieran biopsias gástricas según el protocolo endoscópico de Sídney.

Se consideraron como criterios de inclusión a los pacientes mayores de 18 años sin diferencia de género, a quienes se les haya realizado una endoscopía digestiva alta electiva con toma de biopsias gástricas y datos completos en la ficha de recolección.

Se excluyeron las muestras de gastrectomías y polipectomías, las biopsias de la unión gastroesofágica, las biopsias gástricas con diagnóstico de tumoración, los pacientes con diagnóstico anterior de neoplasia gástrica, con antecedente de gastrectomía, con el procedimiento endoscópico incompleto y las biopsias gástricas tomadas sin cumplir el protocolo de Sídney.

Los datos recolectados fueron ingresados en una base de datos en Microsoft Excel 15.0. Luego fueron analizados mediante el programa STATA v.11. Las variables cuantitativas se expresaron como la media \pm desviación estándar (DE) o medianas y rangos según su distribución, mientras que las variables cualitativas se expresaron en porcentajes (\%).

Sobre las variables, el OLGA es un instrumento para evaluar y determinar la extensión de la atrofia gástrica en 5 estadios que van del 0 al IV por criterios de gravedad. ${ }^{20}$ Los estadios avanzados tienen un mayor riesgo de cáncer gástrico por lo que poseen un valor pronóstico. El OLGIM sustituye la atrofia gástrica por la metaplasia intestinal en la estadificación de la gastritis. ${ }^{24}$

Para el diagnóstico, el antro y el ángulo se consideraron juntos como representativos de la mucosa gástrica distal (no oxíntica), lo que nos da la puntuación del antro, y el cuerpo mayor y la curvatura menor se consideraron juntos como representativos de la mucosa gástrica oxíntica, lo que nos dio la puntuación del cuerpo. La combinación de la puntuación del antro y cuerpo para la GA dio como resultado la puntuación de la etapa de gastritis OLGA, y una combinación de las puntuaciones de MI dio como resultado la puntuación de estadificación del OLGIM.
Durante el procedimiento, se evaluó el patrón endoscópico de la mucosa. Se tomaron biopsias según el protocolo de Sídney: dos muestras en el antro, la primera hacia la curvatura menor, a 2-3 centímetros del píloro; la segunda hacia la curvatura mayor, a 2-3 centímetros del píloro; una muestra en incisura angularis; dos muestras en el cuerpo, la primera hacia la curvatura menor, a 4 centímetros proximal a la incisura angularis y la segunda en el cuerpo medio, hacia la curvatura mayor, a 8 centímetros del cardias. ${ }^{13,14}$

Se analizaron las variables independientes con la variable dependiente usando las pruebas de chi cuadrado para variables cualitativas y prueba $T$ de Student para variables cuantitativas. El nivel de significación establecido fue de $p<0,05$ considerándose un intervalo de confianza del $95 \%$. Finalmente, se realizó un análisis bivariado y una regresión logística considerándose significativo un $p<0,05$ para el análisis de los factores asociados.

Para analizar la concordancia entre los scores del OLGA y OLGIM se usó el coeficiente Kappa de Cohen con un nivel de significación establecido de $p<0,05$ considerándose un intervalo de confianza del $95 \%$.

\section{Resultados}

Con respecto a las características sociodemográficas de la población, la edad media de los 197 sujetos incluidos fue de 54,8 \pm 13,9 años y el 28,7\% era varón. La tabla 1 resume las características básicas y clínico-patológicas. El 86,8\% del total presentó estadios del OLGA/ OLGIM 0-I. La tasa de infección por el $H$. pylori fue del $56,85 \%$.

Los sujetos positivos al $H$. pylori no tuvieron distribuciones de estadios del OLGA y OLGIM con frecuencias mayores en comparación con los sujetos negativos al H. pylori.

La tabla 2 muestra la distribución de la etapa OLGA según la edad. El porcentaje de sujetos con el OLGA en estadio III o IV fue del 5,5\% (2/36) en sujetos con el H. pylori positivo y del 5,3\% (8/150) en sujetos con el H. pylori negativo. Asimismo, el 69,4\% (25/36) de los sujetos con el $H$. pylori positivo fue OLGA estadio 0 y el $64,6 \%(97 / 150)$ fue etapa 0 en sujetos con el $H$. pylori negativo.

En general, se observó un cambio hacia una etapa del OLGA más alta en los sujetos con edades más avanzadas $(p<0,001)$. De acuerdo con la presencia del $H$. pylori, la distribución del estadio no fue significativamente mayor a medida que aumentaba la edad tanto en los sujetos positivos al $H$. pylori como en los sujetos negativos al $H$. pylori. Todos los sujetos menores de 20 años tenían el OLGA en la etapa 0 o I. 
Tabla 1. Características sociodemográficas y clinicas

\begin{tabular}{|c|c|c|c|}
\hline & $\begin{array}{c}\text { Total } \\
(n=197)\end{array}$ & $\begin{array}{c}\text { H. pylori }(+) \\
(\mathrm{n}=112)\end{array}$ & $\begin{array}{l}\text { H. pylori (-) } \\
\quad(n=85)\end{array}$ \\
\hline $\begin{array}{l}\text { Media de edad } \\
\text { (años } \pm D E \text { ) }\end{array}$ & $54,7 \pm 13,9$ & $56,4 \pm 13,5$ & $52,5 \pm 14,3$ \\
\hline Sexo femenino $\mathrm{n}(\%)$ & $139(71,3)$ & $77(55,4)$ & $62(44,6)$ \\
\hline Tabaco n (\%) & $8(4)$ & $5(62,5)$ & $3(37,5)$ \\
\hline Alcohol n (\%) & $15(7,7)$ & $8(53,3)$ & $7(46,7)$ \\
\hline $\begin{array}{l}\text { Infección previa por } \\
\text { el H. pylori n (\%) }\end{array}$ & $38(19,4)$ & $23(60,5)$ & $15(39,5)$ \\
\hline $\begin{array}{l}\text { Antecedente familiar } \\
\text { de CG } n(\%)\end{array}$ & $24(12,2)$ & $14(58,3)$ & $10(41,7)$ \\
\hline \multicolumn{4}{|c|}{ Motivo de la endoscopía n (\%) } \\
\hline Dispepsia & $161(80,5)$ & $92(57,1)$ & $69(42,9)$ \\
\hline Ulcera péptica & $13(7,5)$ & $9(69,2)$ & $4(30,8)$ \\
\hline ERGE & $11(5,5)$ & $2(40)$ & $3(60)$ \\
\hline Sospecha NM & $9(4,5)$ & $5(55,6)$ & $4(44,4)$ \\
\hline Despistaje & $2(1)$ & $1(50)$ & $1(50)$ \\
\hline Otros & $4(2)$ & $3(75)$ & $1(25)$ \\
\hline \multicolumn{4}{|l|}{ Estadio del OLGA n (\%) } \\
\hline 0 & $128(65)$ & $73(57)$ & $55(43)$ \\
\hline I & $30(15,2)$ & $17(56,7)$ & $13(43,3)$ \\
\hline II & $26(13,2)$ & $15(57,7)$ & $11(42,3)$ \\
\hline III & $11(5,6)$ & $6(54,5)$ & $5(45,5)$ \\
\hline IV & $2(1)$ & $1(50)$ & $1(50)$ \\
\hline \multicolumn{4}{|l|}{ Estadio del OLGIM n (\%) } \\
\hline 0 & $115(58,4)$ & $65(56,5)$ & $50(43,5)$ \\
\hline I & $56(28,4)$ & $30(53,6)$ & $26(46,4)$ \\
\hline II & $15(7,6)$ & $12(80)$ & $3(20)$ \\
\hline III & $8(4)$ & $4(50)$ & $4(50)$ \\
\hline IV & $3(1,5)$ & $1(33,3)$ & $2(66,7)$ \\
\hline $\begin{array}{l}\text { El OLGA de alto } \\
\text { riesgo (III-IV) }\end{array}$ & $13(6,6)$ & $7(53,9)$ & $6(46,1)$ \\
\hline $\begin{array}{l}\text { El OLGIM de alto } \\
\text { riesgo (III-IV) }\end{array}$ & $11(5,6)$ & $5(45,5)$ & $6(54,5)$ \\
\hline
\end{tabular}

Tabla 2. Distribución de los estadios del OLGA por edad

\begin{tabular}{ll}
\hline Estadio & Edad \\
OLGA & $p$
\end{tabular}

$<20 \quad 20-29 \quad 30-39 \quad 40-49 \quad 50-59 \quad 60-69 \quad 70-79 \quad 80+$

$\begin{array}{lrrrrrrrrr}\text { H. pylori }(+)(\mathrm{n}=36) & & & & & & & & \\ \mathbf{0} & 0 & 1 & 2 & 5 & 6 & 8 & 2 & 1 & \\ \text { I } & 0 & 0 & 1 & 0 & 2 & 1 & 0 & 0 & \\ \text { II } & 0 & 0 & 1 & 1 & 0 & 0 & 3 & 0 & 0,499 \\ \text { III } & 0 & 0 & 0 & 0 & 1 & 1 & 0 & 0 & \\ \text { IV } & 0 & 0 & 0 & 0 & 0 & 0 & 0 & 0 & \end{array}$

\begin{tabular}{|c|c|c|c|c|c|c|c|c|c|}
\hline \multicolumn{10}{|c|}{ H. pylori $(+)(n=150)$} \\
\hline 0 & 1 & 5 & 11 & 26 & 25 & 26 & 3 & 0 & \\
\hline I & 0 & 2 & 1 & 0 & 10 & 3 & 6 & 2 & \\
\hline II & 0 & 1 & 0 & 3 & 4 & 5 & 4 & 3 & 0,001 \\
\hline III & 0 & 0 & 0 & 2 & 0 & 2 & 2 & 0 & \\
\hline IV & 0 & 0 & 0 & 0 & 0 & 0 & 2 & 0 & \\
\hline \multicolumn{10}{|c|}{ Total $(n=186)$} \\
\hline 0 & 1 & 6 & 13 & 31 & 31 & 34 & 5 & 1 & \\
\hline I & 0 & 2 & 2 & 0 & 12 & 4 & 6 & 2 & \\
\hline ॥ & 0 & 1 & 2 & 4 & 4 & 5 & 7 & 3 & 0,001 \\
\hline III & 0 & 0 & 0 & 2 & 1 & 3 & 2 & 0 & \\
\hline IV & 0 & 0 & 0 & 0 & 0 & 0 & 2 & 0 & \\
\hline
\end{tabular}

La proporción de estadios del OLGA de alto riesgo fue de 13/197 (6,6\%). Las etapas del OLGA de alto riesgo aumentaron significativamente a medida que se acrecentaba la edad en los sujetos tanto del $H$. pylori positivo como del $H$. pylori negativo, aunque esto era más significativo en los sujetos negativos para el $H$. pylori. No se encontró el OLGA de alto riesgo antes de los 40 años, independientemente de la presencia del $H$. pylori, pero aumentó a $16,2 \%, 10,3 \%, 17,3 \%$ y $40,8 \%$ en los sujetos en la cuarta, quinta, sexta y séptima década de vida respectivamente. El OLGIM de alto riesgo mostró una tendencia similar: $0 \%$ antes de los 40 años y hasta $22,6 \%$ en las personas de 70 años.

En cuanto al OLGIM, 11/197 (5,6\%) sujetos fueron considerados de alto riesgo. La distribución de las etapas del OLGIM también aumentó por la edad $(p<0,001)$ (Tabla 3). Todos los pacientes antes de los 40 años fueron OLGIM 0-I. Se observó un estadio avanzado en el $3,2 \%(1 / 31)$ en la cuarta década, en el 2,7\% (1/36) en la sexta década y en el $11,7 \%(2 / 17)$ en la séptima década de la vida. 
Tabla 3. Distribución de los estadios del OLGIM

\begin{tabular}{ll}
\hline Estadio & Edad \\
OLGIM & $p$ \\
\hline
\end{tabular}

$<20 \quad 20-29 \quad 30-39 \quad 40-49 \quad 50-59 \quad 60-69 \quad 70-79 \quad 80+$

$\begin{array}{llllllllll}\text { H. pylori }(+)(n=36) & & & & & & & \\ 0 & 0 & 1 & 2 & 5 & 6 & 8 & 1 & 1 & \\ \text { I } & 0 & 0 & 2 & 0 & 2 & 1 & 3 & 0 & \\ \text { II } & 0 & 0 & 0 & 1 & 0 & 1 & 1 & 0 & 0,641 \\ \text { III } & 0 & 0 & 0 & 0 & 1 & 0 & 0 & 0 & \\ \text { IV } & 0 & 0 & 0 & 0 & 0 & 0 & 0 & 0\end{array}$

$\begin{array}{llllllllll}\text { H. pylori }(-)(\mathrm{n}=150) & & & & & & & \\ \mathbf{0} & 1 & 5 & 10 & 25 & 22 & 22 & 2 & 0 & \\ \text { I } & 0 & 3 & 3 & 4 & 13 & 10 & 9 & 3 & \\ \text { II } & 0 & 0 & 0 & 1 & 4 & 3 & 1 & 2 & 0,001 \\ \text { III } & 0 & 0 & 0 & 1 & 0 & 1 & 2 & 0 & \\ \text { IV } & 0 & 0 & 0 & 0 & 0 & 0 & 3 & 0\end{array}$

Total $(n=186)$

$\begin{array}{llllllllll}\mathbf{0} & 1 & 6 & 12 & 30 & 28 & 30 & 3 & 1 & \\ \text { I } & 0 & 3 & 5 & 4 & 15 & 11 & 12 & 3 & \\ \text { II } & 0 & 0 & 0 & 2 & 4 & 4 & 2 & 2 & 0,001 \\ \text { III } & 0 & 0 & 0 & 1 & 1 & 1 & 2 & 0 & \\ \text { IV } & 0 & 0 & 0 & 0 & 0 & 0 & 3 & 0 & \end{array}$

$\mathrm{Al}$ analizar la edad con el desarrollo de los estadios de alto riesgo del OLGA/OLGIM, se estableció que tener una edad mayor a 60 años está relacionado a tener un estadio de alto riesgo. En términos de edad, calculamos los OR para las etapas del OLGA de alto riesgo en los sujetos en sus 40, 50 y 60 en comparación con los menores de 40 años, porque ningún sujeto de entre 20 y 30 años tenía una etapa de alto riesgo. En el modelo de regresión logística univariante, la edad ( $\geq 40$ años) no se asoció significativamente con las etapas del OLGA ni del OLGIM de alto riesgo.

El 79,6\% (157/197) presentó estadios no avanzados del OLGA/OLGIM. La distribución del estadio del OLGA se correlacionó positivamente con el estadio del OLGIM (coeficiente de correlación: 0,73; $p<0,001$ ) (Tabla 4).
Tabla 4. Concordancia entre el OLGA y el OLGIM

\begin{tabular}{|c|c|c|c|c|c|c|c|}
\hline & & \multicolumn{5}{|c|}{ Estadios del OLGA } & \multirow[t]{2}{*}{ Total } \\
\hline & & 0 & I & II & III & IV & \\
\hline \multirow{5}{*}{$\begin{array}{l}\text { Estadios } \\
\text { del OLGIM }\end{array}$} & 0 & 115 & 0 & 0 & 0 & 0 & 115 \\
\hline & I & 12 & 30 & 12 & 2 & 0 & 56 \\
\hline & ॥ & 1 & 0 & 13 & 1 & 0 & 15 \\
\hline & III & 0 & 0 & 0 & 8 & 0 & 8 \\
\hline & IV & 0 & 0 & 1 & 0 & 2 & 3 \\
\hline Total & & 128 & 30 & 26 & 11 & 2 & 197 \\
\hline
\end{tabular}

Kappa: 0,739 .

\section{Discusión}

La inflamación crónica de la mucosa gástrica puede desencadenar una cascada de cambios histológicos que eventualmente puede resultar en un CG. ${ }^{7-12}$ El CG se clasifica histopatológicamente como un carcinoma de tipo intestinal y difuso según Lauren. ${ }^{7,8}$ La presencia de la GA, la MI y la displasia son factores de riesgo importantes para el tipo intestinal y a esta secuencia de lesiones premalignas se la denomina cascada de Pelayo Correa. ${ }^{7-12,18,19}$

Cuando la gastritis es severa y extensa genera un estado de hipoclorhidria que favorece la sobrepoblación bacteriana con altas concentraciones de nitritos; los cuales, en combinación con las aminas y amidas, forman compuestos $\mathrm{N}$-nitroso con alta potencialidad carcinogénica. ${ }^{9}$ Existen factores de riesgo ambientales asociados al CG: el tabaco, el alcohol, la obesidad, las dietas ricas en sal y carnes procesadas y la infección por el $H$. pylori. Este último es el factor de riesgo más importante para el desarrollo de las lesiones premalignas. ${ }^{1-5,18-20} \mathrm{El}$ efecto de erradicar el H. pylori sobre la GA y la MI es incierto; sin embargo, se plantea una regresión lenta de la GA, pero la regresión de la MI parece menos probable. ${ }^{24,25}$

La división histológica para biopsias gástricas más utilizada es el sistema de Sídney modificado, cuyo reporte final combina el tipo, la intensidad y la extensión de la patología gástrica además de la posible etiología. ${ }^{13,14}$ Asimismo, establece un protocolo que señala donde deben ser tomadas las biopsias: dos muestras en el antro, la primera hacia la curvatura menor, a 2-3 centímetros del píloro, la segunda hacia la curvatura mayor, a 2-3 centímetros del píloro; una muestra en la incisura angular, dos muestras en el cuerpo, la primera hacia la curvatura menor, a 4 centímetros proximal a la incisura angularis, y la segunda en cuerpo medio, hacia la curvatura mayor, 
a 8 centímetros del cardias. ${ }^{13,14}$ Por otro lado, provee una escala visual análoga para clasificar el grado de inflamación, atrofia, metaplasia y la densidad del H. pylori. ${ }^{13,14,18}$ La nomenclatura para la gastritis sigue siendo inconsistente a pesar de que existen métodos como este, ${ }^{18,20}$ debido a que los resultados carecen de valor pronóstico. Aún no se logra identificar candidatos para una vigilancia endoscópica adecuada. ${ }^{15}$

En el año 2005, el grupo internacional Operative Link on Gastritis Assessment (OLGA) planteó un nuevo sistema como instrumento para evaluar y determinar la extensión de la atrofia y la metaplasia intestinal. ${ }^{15,}{ }^{16,20}$ La GA se define como la pérdida de las glándulas apropiadas. Esto ocurre cuando son reemplazadas por fibrosis o estructuras glandulares de localización inapropiada, fenómeno también denominado metaplasia. ${ }^{22}$ Está demostrado que a mayor extensión de la GA hay un mayor riesgo de desarrollar un CG. ${ }^{10-12,22}$

El método del OLGA clasifica a la GA en 5 estadios que van del 0 al IV, donde 0 es no atrofia, I, atrofia mínima, y IV, el grado más severo. ${ }^{20}$ Está descripto que los pacientes con estadios avanzados (III y IV) tienen mayor riesgo de tener CG por lo que este sistema sí posee un valor pronóstico que resulta interesante para generar estrategias de seguimiento en los grupos de riesgo. ${ }^{15-20}$ Por la variabilidad interobservador en el diagnóstico de la GA, se ha sugerido reemplazar la GA por la MI (sistema del OLGIM) aunque esto pudiera disminuir levemente la sensibilidad. $^{24}$

El OLGA y el OLGIM se utilizan como posibles sistemas de estadificación histológica para la evaluación del riesgo del CG y han despertado un gran interés en el cribado y su vigilancia. ${ }^{24,29}$ Varios estudios, aunque pequeños y controvertidos, han demostrado el valor de las etapas del OLGA y OLGIM de alto riesgo en la estratificación del riesgo del CG. ${ }^{24,29}$ Así tenemos que Yue et al. revelaron en un metaanálisis que la etapa III / IV del OLGA o OLGIM estaba asociada con un mayor riesgo de neoplasia. ${ }^{29}$

En un estudio de casos y controles de Martínez et al. sólo el $12,3 \%$ tenía OLGA III/IV y concluye que el OLGA permite detectar un $61,8 \%$ más de atrofia que la detectada con un muestreo insuficiente de biopsias gástricas. ${ }^{30}$ Con respecto al $H$. pylori, la prevalencia de la infección fue similar en ambos grupos: $60 \%$ y $57 \%$ respectivamente. ${ }^{30}$ En Holanda, un estudio de cohorte mostró una incidencia anual del $0,1 \%$ para la GA;
$0,25 \%$ para la MI; $0,6 \%$ para la displasia leve a moderada y $6 \%$ para la displasia severa. ${ }^{26}$

Rugge et al. estudiaron a 439 sujetos con dispepsia, en quienes se realizó una biopsia de acuerdo con el protocolo de Sídney y la estadificación con el OLGA y encontraron 21 casos en los estadios III y IV (4,8\%), una proporción similar al estudio de Ramírez Mendoza et al. (4,78\% vs. $2,78 \%$ respectivamente). ${ }^{15,22}$

Slama et al. realizaron un estudio descriptivo de $100 \mathrm{ca}-$ sos de gastritis crónica por el $H$. pylori y utilizaron los OLGA y OLGIM para establecer estadios de atrofia y metaplasia. Revelaron, al igual que nuestros datos, una correlación significativa entre la edad y la estadificación del OLGA. ${ }^{27}$ Además, mostraron una correlación altamente positiva significativa entre ambos sistemas, dato que nuestro estudio también encontró. ${ }^{27}$

Asimismo, Ji Hyung Nam et al. estudiaron a 632 pacientes para evaluar la distribución de estadios del OLGA y OLGIM según la edad y el estado del H. pylori en la población coreana y determinaron que los sujetos con el $H$. pylori positivo tuvieron distribuciones de estadios del OLGA y OLGIM significativamente más altas en comparación con los sujetos con $H$. pylori negativo (ambos $p<0,001) .{ }^{28}$ En nuestro estudio no se halla una asociación significativa entre los estadios avanzados del OLGA/OLGIM con la presencia del H. pylori. Esto podría explicarse por la poca cantidad de pacientes hallados en estos estadios. Sin embargo, la tasa del $H$. pylori fue del $59 \%$ en este estudio, ${ }^{28}$ casi similar a la nuestra del $56,85 \%$. En dicho trabajo el porcentaje de sujetos con estadio del OLGA III o IV fue del 3,5\% (9/259) en los sujetos con el $H$. pylori negativo y del 25,7\% (96/373) en los sujetos con el $H$. pylori positivo, a diferencia de nuestro estudio que tiene porcentajes similares en ambos subgrupos: $5,55 \%$ (2/36) en los $H$. pylori positivo y 5,33\% (8/150) en los H. pylori negativo. Nosotros no logramos asociar el tabaquismo y la infección por el $H$. pylori con las etapas del OLGA y OLGIM de alto riesgo a diferencia del trabajo de Nam et al. ${ }^{28}$

En un estudio peruano de Liu Bejarano, los estadios avanzados del OLGA se asociaron a las lesiones concomitantes de displasia y al CG temprano y avanzado; mientras los estadios 1 y 2 se asociaron a lesiones inflamatorias y benignas. ${ }^{31}$ Nosotros no encontramos relación entre el OLGA/OLGIM de alto riesgo con lesiones neoplásicas, probablemente por contar con una muestra reducida. Asimismo, Yun et al. demostraron en Corea que los estadios 
del OLGA/OLGIM de alto riesgo son marcadores de predicción importantes para el CG independientemente de la infección por el $H$. pylori o los antecedentes familiares de CG, no solo para el tipo intestinal, sino también para el CG de tipo difuso. ${ }^{32}$

El presente estudio tiene dos principales limitaciones. La primera es que se realizó en un solo centro; , por lo tanto, no podemos excluir la posibilidad de un sesgo de selección. En segundo lugar, contamos con un tamaño de muestra reducido.

En conclusión, los casos del OLGA/OLGIM de alto riesgo son infrecuentes antes de los 40 años y aumentan significativamente con la edad. No se evidenció una relación con la presencia del $H$. pylori. En nuestro país es la primera vez que se realiza un estudio que determine la presencia de lesiones premalignas gástricas mediante estos métodos histológicos y su relación con la edad. Nos parece importante, por un lado, identificar a la población de riesgo, ya que nos ayuda a realizar un seguimiento endoscópico cercano y adecuado para prevenir la aparición del CG. Por otro lado, según nuestros datos, sólo el 13,19\% de los pacientes necesitaría endoscopías de control. De esta manera, se evitarían gastos por la repetición de estudios innecesarios en la mayoría de los pacientes. Se requieren de estudios con un mayor número de población y multicéntricos que permitan obtener resultados extrapolables a nuestra población.

\section{Conflictos de intereses. Los autores declaran no tener nin- gún conflicto de interés.}

\section{Aviso de derechos de autor}

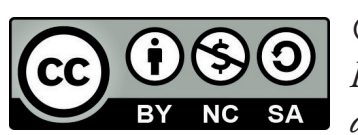

(C) 2021 Acta Gastroenterológica Latinoamericana. Este es un artículo de acceso abierto publicado bajo los términos de la Licencia Creative Commons Attribution (CC BY-NC-SA 4.0), la cual permite el uso, la distribución y la reproducción de forma no comercial, siempre que se cite al autor y la fuente original.

Cite este artículo como: Carlin Ronquillo A, Ventura León A, Espinoza Ríos JL y col. Distribución de estadios de OLGA y OLGIM según edad y estado del Helicobacter pylori en un hospital público nivel III en Lima, Perú. Acta Gastroenterol Latinoam. 2021;51(1):76-83. https://doi. org/10.52787/uccm 2971

\section{Referencias}

1. Ferlay J, Soerjomataram I, Dikshit R, Eser S, Mathers C, Rebelo M, Parkin DM, Forman D, Bray F. Cancer incidence and mortality worldwide: sources, methods and major patterns in GLOBOCAN 2012. International Journal of Cancer. 2015;136(5):E359-86.

2. Ikeda Y, Mori M, Kamakura T, Haraguchi Y, Saku M, Sugimachi K. Improvements in diagnosis have changed the incidence of histological types in advanced gastric cancer. $\mathrm{Br} \mathrm{J}$ Cancer. 1995;72(2):424-6.

3. Ministerio de Salud del Perú, Instituto Nacional de Enfermedades Neoplásicas, Departamento de Epidemiología y Estadística del Cáncer. Registro de Cáncer de Lima Metropolitana 20042005. 2013; IV.

4. Pasechnikov V, Chukov S, Fedorov E, Kikuste I, Leja M. Gastric cancer: Prevention, screening and early diagnosis. World J Gastroenterol. 2014;20(38):13842-62.

5. Marcos-Pinto R, Carneiro F, Dinis-Ribeiro M, Wen X, Lopes C, Figueiredo C, Machado JC, Ferreira RM, Reis CA, Ferreira J, Pedroto I, Areias J. First-degree relatives of patients with early-onset gastric carcinoma show even at young ages a high prevalence of advanced OLGA/OLGIM stages and dysplasia. Aliment Pharmacol Ther. 2012;35(12):1451-9.

6. Correa P. Gastric cancer: two epidemics? Dig Dis Sci. 2011;56(5):1585-6.

7. Lauren P. The two histological main types of gastric carcinoma: diffuse and so-called intestinal-type carcinoma. An attempt at a histo-clinical classification. Acta Pathol Microbiol Scand. 1965;64:31-49.

8. Capelle L, de Vries AC, Haringsma J, Ter Borg F, de Vries RA, Bruno MJ, van Dekken H, Meijer J, van Grieken NCT, Kuipers EJ. The staging of gastritis with the OLGA system by using intestinal metaplasia as an accurate alternative for atrophic gastritis. Gastrointestinal Endoscopy. 2010;71(7):1150-8.

9. Correa P. Human gastric carcinogenesis: a multistep and multifactorial process-First American Cancer Society Award Lecture on Cancer Epidemiology and Prevention. Cancer Research. 1992;52(24):6735-40.

10. Ohata H, Kitauchi S, Yoshimura N, Mugitani K, Iwane M, Nakamura H, Yoshikawa A, Yanaoka K, Arii K, Tamai H, Shimizu Y, Takeshita T, Mohara O, Ichinose M. Progression of chronic atrophic gastritis associated with Helicobacter pylori infection increases risk of gastric cancer. Int J Cancer. 2004;109:138-43.

11. Tatsuta M, Iishi H, Nakaizumi A, Okuda S, Taniguchi H, Hiyama T, Tsukuma H, Oshima A. Fundal atrophic gastritis as a risk factor for gastric cancer. Int J Cancer. 1993;53:70-4.

12. Rugge M, Genta RM, OLGA Group. Staging gastritis: an international proposal. Gastroenterology. 2005;129(5):1807-8.

13. Price AB, Misiewicz JJ. Sydney classification for gastritis. Lancet. 1991;337(8734):174.

14. Dixon MF, Genta RM, Yardley JH, Correa P. Classification and grading of gastritis. The updated Sydney System. International Workshop on the Histopathology of Gastritis, Houston 1994. Am J Surg Pathol. 1996;20(10):1161-81.

15. Ramírez-Mendoza P, Ruiz-Castillo SA, Maroun-Marun C, Trujillo Benavides O, Baltazar-Montúfar P, Méndez del Monte R, Ángeles-Garay U. Estadificación de la gastritis con el sistema OLGA; prevalencia de estadios avanzados de atrofia gástrica en pacientes mexicanos. Rev Gastroenterol Mex. 2011;76(4):302-8. 
16. Rugge M, Fassan M, Pizzi M, Farinati F, Sturniolo GC, Plebani M, Graham DY. Operative link for gastritis assessment vs operative link on intestinal metaplasia assessment. World J Gastroenterol. 2011;17(41):4596-601.

17. Ramírez-Mendoza P, González-Angulo J, Ángeles-Garay U, Segovia-Cueva GA. Evaluación histopatológica de gastritis atrófica. Comparación de los sistemas Sídney y OLGA. Rev Med Inst Mex Seguro Soc. 2008;46(2):135-9.

18. Park YH, Kim N. Review of Atrophic Gastritis and Intestinal Metaplasia as a Premalignant Lesion of Gastric Cancer. J Cancer Prev. 2015;20(1):25-40.

19. El-Zimaity H. Gastritis and Gastric Atrophy. Curr Opin Gastroenterol. 2008;24(6):682-6.

20. Rugge M, Correa P, Di Mario F, El-Omar E, Fiocca R, Geboes K, Genta RM, Graham DY, Hattori T, Malfertheiner P, Nakajima S, Sipponen P, Sung J, Weinstein W, Vieth M. OLGA staging for gastritis: A tutorial. Digestive and Liver Disease. 2008;40(8):650-8.

21. Rugge M, de Boni M, Pennelli G, de Bona M, Giacomelli L, Fassan M, Basso D, Plebani M, Graham DY. Gastritis OLGA-staging and gastric cancer risk: a twelve-year clinico-pathological follow-up study. Aliment Pharmacol Ther. 2010;31(10):1104-11.

22. Rugge M, Meggio A, Pennelli G, Piscioli F, Giacomelli L, De Pretis G, Graham DY. Gastritis staging in clinical practice: the OLGA staging system. Gut. 2007;56(5):631-6.

23. Venerito M, Malfertheiner P. Preneoplastic Conditions in the Stomach: Always a Point of No Return?. Dig Dis. 2015;33:5-10.

24. Rollán A, Cortés P, Calvo A, Araya R, Bufadel ME, González R, Heredia C, Muñoz P, Squella F, Nazal R, Gatica MA, Gobelet J, Estay R, Pisano R, Contreras L, Osorio I, Estela R, Fluxá F, Parra-Blanco A. Diagnóstico precoz de cáncer gástrico. Propuesta de detección y seguimiento de lesiones premalignas gástricas: protocolo ACHED. Rev Med Chile. 2014;142(9):1181-92.
25. Wang J, Xu L, Shi R, Huang X, Li SWH, Huang Z, Zhang G. Gastric atrophy and intestinal metaplasia before and after Helicobacter pylori eradication: a meta-analysis. Digestion. 2011;83(4):253-60.

26. De Vries AC, van Grieken NCT, Looman CWN, Casparie MK, de Vries E, Meijer GA, Kuipers EJ. Gastric cancer risk in patients with premalignant gastric lesions: a nationwide cohort study in the Netherlands. Gastroenterology. 2008;134(4):945-52.

27. Slama SB, Ghachem DB, Dhaoui A, Taieb Jomni M, Hédi Dougui M, Bellil K. Helicobacter pylori gastritis: assessment of OLGA and OLGIM staging systems. Pan African Medical Journal. 2016;23:28.

28. Nam JH, Choi IJ, Kook MC, Lee JY, Cho SJ, Nam SY, Kim CG. OLGA and OLGIM Stage Distribution According to Age and Helicobacter pylori Status in the Korean Population. Helicobacter. 2014;19(2):81-9.

29. Yue H, Shan L, Bin L. The significance of OLGA and OLGIM staging systems in the risk assessment of gastric cancer: a systematic review and meta-analysis. Gastric Cancer. 2018;21(4):579-87.

30. Martínez D, Otero W, Ricaurte O. Impacto del sistema OLGA en la detección de gastritis crónica atrófica en Colombia: un estudio de casos y controles. Rev Col Gastroenterol. 2016;31(4):360-7.

31. Liu Bejarano H. Gastritis crónica atrófica: concordancia endoscópica, histológica, lesiones asociadas y aplicación de la cromoendoscopia virtual. Rev Gastroenterol Perú. 2011; 31(2):116-23.

32. Yun CY, Kim N, Lee J, Lee JY, Hwang YJ, Lee HS, Yoon H, Shin CM, Park YS, Kim JW, Lee DH. Usefulness of OLGA and OLGIM system not only for intestinal type but also for diffuse type of gastric cancer, and no interaction among the gastric cancer risk factors. Helicobacter. 2018;23(6):e12542. 